\title{
Chapter 20 \\ Biodiversity and Health in the Face of Climate Change: Perspectives for Science, Policy and Practice
}

\author{
Melissa R. Marselle, Jutta Stadler, Horst Korn, Katherine N. Irvine, \\ and Aletta Bonn
}

\begin{abstract}
Increases in non-communicable diseases, biodiversity loss and climate change are among the greatest global challenges society is facing today. At the same time, biodiverse natural environments can buffer the negative effects of climate change to society and support human health. Contributions in this volume demonstrate the growing interest in the impact of biodiversity on human health and wellbeing in the face of climate change. The chapters in this volume present and critically review the growing body of literature on the associations of biodiversity and human health, with mounting evidence of positive effects for physical health and wellbeing. In this concluding chapter, we summarise the key outcomes of the chapters in this book. Synthesising the main results with a link to current policy, we develop
\end{abstract}

\footnotetext{
M. R. Marselle ( $\square)$

Department of Ecosystem Services, Helmholtz Centre for Environmental Research - UFZ, Leipzig, Germany

German Centre for Integrative Biodiversity Research (iDiv) Halle-Jena-Leipzig,

Leipzig, Germany

e-mail: melissa.marselle@ufz.de

J. Stadler $\cdot$ H. Korn

Federal Agency for Nature Conservation (BfN), Isle of Vilm, Putbus, Germany

e-mail: jutta.stadler@bfn.de; horst.korn@bfn.de

K. N. Irvine

Social, Economic and Geographical Sciences Research Group, The James Hutton Institute, Aberdeen, Scotland, UK

e-mail: katherine.irvine@hutton.ac.uk
}
A. Bonn
Department of Ecosystem Services, Helmholtz Centre for Environmental Research - UFZ, Leipzig, Germany
German Centre for Integrative Biodiversity Research (iDiv) Halle-Jena-Leipzig, Leipzig, Germany
Institute of Biodiversity, Friedrich Schiller University Jena, Jena, Germany e-mail: aletta.bonn@ufz.de 
recommendations to address the urgent health and sustainability challenges in science, policy and practice.

Keywords Synthesis $\cdot$ Biodiversity $\cdot$ Climate change adaptation $\cdot$ Noncommunicable disease $\cdot$ Policy recommendations $\cdot$ Science-policy interface

\section{Highlights}

- Contributions in this volume present growing evidence of the linkages between biodiversity and physical, mental and spiritual aspects of health and well-being.

- Evidence seems to suggest strong links between biodiversity and physical health and well-being, which points to important avenues for health treatments and natural resource management.

- Currently disjointed policy sectors of biodiversity conservation and management, public health and climate change need to work together to foster the foundation of our society - considering the ecosystem and human health in a One Health agenda.

- Arguing for health as a central benefit to society that results from nature conservation and good biodiversity management should improve the public and political interest in the subject.

- Evidence is sufficient to implement 'no regret' actions now that are mainly based on nature-based solutions.

- Key steps to integrate considerations of biodiversity, public health and climate change into research, policy and management agendas are provided.

\subsection{Introduction}

The rise in non-communicable diseases (World Health Organization [WHO] 2017a, b) combined with biodiversity loss and climate change (Bellard et al. 2012; Steffen et al. 2015) are among the greatest global challenges society is facing today. While biodiversity provides the foundation for human well-being, human societies also provide the greatest drivers for biodiversity loss and climate change. Central to addressing these critical challenges are the questions ' How does biodiversity matter for human health and well-being?' and 'What implications does this have for efforts to address our current predicament?' (WHO and CBD 2015). Increasingly, science is starting to unravel relationships of how biodiversity impacts human health and well-being, and we are at the beginning of an exponential rise in research activity, as shown by the contents of this book. The chapters critically review the growing body of literature that examines biodiversity's contribution to physical health as well as mental and spiritual well-being in the face of climate change. In their totality, these chapters encompass the mounting evidence of positive effects on physical health and well-being (see Lindley et al. Chap. 2, Dadvand et al. Chap. 6, Cook et al. Chap. 11, Hunter et al. Chap. 17, this volume). Some effects on physical health 
related to physical activity and obesity remain inconclusive, possibly because of study design and confounding variables such as socio-economic factors, which need to be considered carefully when designing studies (Kabisch Chap. 5, this volume). Some specific biodiversity-health associations are negative, especially with regard to allergies and vector-borne diseases (Damialis et al. Chap. 3, Müller et al. Chap. 4, this volume), whereas effective spatial planning and management actions can mitigate these effects (Elmqvist et al. Chap. 18, Heiland et al. Chap. 19, this volume). There is also some evidence of the positive effects of biodiversity on mental health and well-being (Marselle et al. Chap. 9, this volume). Importantly, this volume also considers spiritual well-being (Irvine et al. Chap. 10, this volume), which to date has been subject to little attention. Management of biodiversity could therefore form a globally important natural health service.

By reviewing and synthesising the available literature and recent findings, the authors in this volume develop an evidence base for how biodiversity can contribute to physical, mental and spiritual aspects of health and well-being. Importantly, the volume starts to further develop the theory of biodiversity-health relationships (Marselle Chap. 7, this volume), a necessary component for future studies. The authors identify the different mechanisms for biodiversity-health pathways by building on existing work (e.g. Hartig et al. 2014; Markevych et al. 2017; Potschin and Haines-Young 2011; van den Bosch and Ode Sang 2017). As this is an emerging research area, it was at times challenging for some chapters to relate to primary data and analyses where all three topics - biodiversity, health and climate change were assessed together. Similarly, while the contributions draw on expertise from different disciplines, an additional challenge encountered was moving beyond analyses of green space in general to focus on the specific contribution of biodiversity in particular, for which few studies exist to date, although research has grown in the past decade (e.g. Aerts et al. 2018; Fuller et al. 2007; Dallimer et al. 2012; Lovell et al. 2014). To foster further research, the chapters identify knowledge gaps and areas for new research avenues as well as improvement through enhanced or better aligned indicators and metrics (de Vries \& Snep, Chap. 8, this volume). These metrics should also link to existing policy targets in public health and nature conservation (Davies et al. Chap. 12, Korn et al. Chap.14, MacKinnon et al. Chap. 16, this volume). We need to move further in our research efforts to quantify the benefits and risks that biodiversity provides for human health, and how interaction with plants and animals shapes our physical, mental and spiritual health and well-being as well as societal and cultural practices. Studies have started to explore dose-response relationships of nature and health (e.g. Cox et al. 2017; Shananhan et al. 2015), which need to be further expanded in order to foster our understanding of the impact of duration and exposure of contact with biodiversity on health. This knowledge is required to aid development of 'health treatments', both through natural resource management interventions via configuration of green and blue spaces and through active social interventions, such as health walks (Marselle et al. 2014; Cook et al. Chap. 11, MacKinnon et al. Chap. 16, Hunter et al. Chap. 17, all this volume). Importantly, unravelling the different mechanisms requires a targeted and innovative study design and consideration of confounding factors to account for different 
configurations of green spaces as well as socioeconomic contexts in different cultural and ethnic settings.

By evaluating a broad range of case studies, the authors also demonstrate how managing green spaces for biodiversity and health can additionally contribute to adapting to the effects of a changing climate (Keune et al. Chap. 15, MacKinnon et al. Chap. 16, this volume), both as communities and as individuals (De Young Chap. 13, this volume). The chapters lay out practical recommendations for policy and practice as well as how to integrate existing knowledge into urban planning and management (Hunter et al. Chap. 17, Elmqvist et al. Chap. 18, Heiland et al. Chap. 19 , this volume). Importantly, proactive planning can contribute actively to the public health agenda (Cook et al. Chap. 11, this volume) and help to increase a city's resilience in the face of climate change (Elmqvist et al. Chap. 18, this volume). Managing green spaces may also alleviate health equity issues (Kabisch Chap. 5, Cook et al. Chap. 11, this volume). In many areas we already know enough to act and to implement 'no regret' actions. It becomes obvious that the global challenges and goals related to biodiversity and health in a changing climate cannot be tackled by one discipline or one sector alone. Informed transdisciplinary dialogue and collaboration is clearly required to address the pressing research questions and to implement actions. The linkages between biodiversity and health are increasingly becoming recognized in both local and regional conservation management and in international policy development (Korn et al. Chap. 14, this volume). Fundamentally, the 2050 Vision of Biodiversity of the Convention of Biological Diversity (CBD) and the United Nations 2030 Agenda for Sustainable Development depend on joint action from many sectors and the alignment of environmental and societal goals. Implementation on the ground will need to be monitored and evaluated for effectiveness (see Hunter et al. Chap. 17, this volume), both for health outcomes and for synergies and trade-offs with conservation and climate policy goals.

Overall, quantifying the health benefits of interventions should also be supported by economic cost-benefit analysis to assess the value and cost-effectiveness of nature-based solution (NBS) measures for health and related co-benefits. These analyses should also support scenario development to assess different future trajectories at regional and global levels to inform decision making in policy and practice. Here, ongoing work with the scenarios and modelling expert group of the Intergovernmental Science-Policy Platform on Biodiversity and Ecosystem Services (IPBES) should pay special attention to the effects of biodiversity scenarios for public health. Finally, coordinated governance systems need to be developed and established to foster the sustainable use and enhancement of biodiversity to promote human health for all people in a changing climate.

In this concluding chapter, we synthesize the main results from the chapters and link them to current policy developments on a European and a global scale. Based on the evidence provided in this volume and drawing from the recommendations of the European Network of Heads of Nature Conservation Agencies (ENCA) derived from the European conference 'Biodiversity and Health in the face of climate change - Challenges, Opportunities and Evidence Gaps' (Marselle et al. 2018), we 
develop recommendations to address the urgent health and sustainability challenges in science, policy and practice.

\subsection{Evidence of Effects of Biodiversity on Physical Health}

Biodiverse natural environments and climate change interact to influence human physical health and well-being in positive and negative ways. Case study examples illustrate these interrelationships for extreme heat, allergenic plants and vector-borne diseases. The effects of nature and health relationships on specific populations groups - children and different socio-economic groups - are highlighted.

There are important links between biodiversity and physical health as demonstrated by the review of Sarah Lindley and co-authors. They argue that both biodiversity and climate change set important boundary conditions for human health, as they influence many elements that impact on health and well-being of individuals, for example through altered ecosystem functions and services. The authors point out that, especially in the face of a changing climate, NBS are needed to adapt to or mitigate negative climate-induced stressors, such as heat waves, reduced air quality, flooding or water quality regulation, that have serious impacts on human health. Many of the available studies are drawn from urban environments where climate effects may be experienced most dramatically due to the urban heat-island effect, and evidenced by the presented case study from Greater Manchester, England. The authors point to the need to consider socio-economic confounding factors to fully address the challenges of understanding the links between biodiversity and physical health, and for research to develop robust metrics and indicators to measure not only the state but also trends of linkages. Overall, it will be important to identify what configurations of green spaces in cities are most beneficial to promote health in order to provide input to urban planning and management.

Athanasios Damialis and co-authors discuss the negative effects of biodiversity and physical health by highlighting the specific issue of pollen allergies. As allergy prevalence has increased worldwide, partly due to a warming climate, their review provides a greater understanding of the emerging challenges. In particular, the authors call for better spatial and temporal risk mapping and forecasting of potential pollen exposure to advise allergy-sensitive individuals as well as to inform urban planning measures to develop green spaces that can minimise allergenic pollen exposure. Importantly, pollen can be a carrier of biochemical complex particles that can additionally affect health, and only recently has an understanding of the pollen microbiome begun to emerge. With the spread of invasive and alien allergenic species in a changing climate, such as ragweed (Ambrosia artemisiifolia), we need to better understand how these distributions can be assessed, predicted and proactively managed. Here, the development of automated, near-real-time pollen 
measurements is exciting and needs operationalisation on a greater scale to provide exposure risk alerts, environmental health-service infrastructure and personalised forecasts.

Another health issue derived from biodiversity-human contacts as discussed by Ruth Müller and co-authors is vector-borne diseases (VBDs), i.e. illnesses caused by parasites, viruses and bacteria transmitted by a vector, often insects, like blood sucking mosquitos. These are estimated to account for $17 \%$ of the global burden of non-communicable diseases, often affecting poorer populations living in degraded ecosystems in the Global South. In Europe, on average 77,000 people are affected by VBDs, and this figure is expected to rise, as abundance and regional distribution of these vectors are shaped by a changing climate as well as human transport. A warming climate may facilitate the spread of alien invasive species such as the Asian tiger mosquito (Aedes albopictus) and disease-transmitting ticks to Northern Europe as well as alter vector and host behaviour. Understanding and forecasting these changes is needed to inform pro-active natural resource management to prevent and halt establishment of vector populations. Notably, the diversity of pathogens as well as the diversity of vectors and hosts are as yet largely unknown and requires further research. Since biodiverse environments can contribute to discovering natural and novel insecticides as well as medially active compounds, global conservation efforts for biodiversity hotspots are needed to maintain options for vector control and pharmaceutical development. In addition, genetic tools in vector control need to be understood and further developed whilst, possibly more importantly, the socio-ecological systems need to be considered and traditional knowledge within local communities incorporated to manage VBDs. As the authors argue, most VBDs can be prevented through vector control if managed effectively, and a transdisciplinary approach across sectors is needed for successful implementation. It is not mainly the lack of scientific expertise but a lack of capacity and capabilities for implementation of good practice that hinder effective management. Comprehensive national strategies, community engagement and the application of varied intervention toolboxes are needed. At a policy level the WHO Global Vector Control Response 2017-2030 provides strategic guidance to deal with VBDs, and should be incorporated in climate adaptation and conservation policies.

Nadia Kabisch highlights in her review that socio-economic and sociodemographic effects are strong co-determinants of health, and confound the association of the impact of green space on health in many studies. Whilst these confounding effects will always be strong predictors, associations between green space and health vary in strength across different case studies and evidence appears strongest with respect to cardiovascular diseases and mental health. As several studies reported a moderating effect of urban green spaces on health inequalities between different socio-economic groups, this points to important avenues for green space urban planning especially in deprived neighbourhoods. The perceived quality and safety of green spaces seems to play a particularly important role in determining actual use of those spaces. Appreciation of different green space qualities varies between age 
groups, and therefore management should focus on providing safe and high-quality green spaces for all members of the community. For future research, the author suggests mixed methods approaches that employ both quantitative and qualitative investigations based on both empirical observations and experimental designs in order to disentangle the determining factors for urban green space and health relationships.

For children, considered as an especially vulnerable group of health beneficiaries, Payam Dadvand and co-authors show that even before birth, prenatal exposure of mothers to green space can improve pregnancy outcomes. Contact with green space may further aid cognitive and behavioural development in children, and has been reported to have some effects on reducing attention-deficit hyperactivity disorder (ADHD) symptoms. The authors identify potential mechanisms including: stress reduction; a higher level of social contacts and increased physical activity; reduction of urban environmental stressors, including noise, heat and air pollution; and increased contact with environmental microbiota. The latter has been shown to increase immunoregulation in several studies. The evidence of the impact of green space contact on respiratory and allergic conditions is inconsistent, as green spaces can provide positive effects whilst they are also a source of fungal spores and pollens. Proactive green space management can help to reduce asthma through careful plant species selection, increasing species diversity and mitigating exposure to air pollution. Despite the opportunity for greater physical activity in green spaces, there was inconsistent evidence of a reduction in obesity and overall increase in physical activity, possibly as studies did not sufficiently account for the quality of green spaces. The authors recommend that investigations should be carefully designed in order to account for confounding factors, such as quality of green space. Overall, in their synthesis, the authors advocate that biodiverse natural areas, especially in urban settings, are important factors for child health and development.

\subsection{Mental Health and Spiritual Well-Being Benefits of Biodiversity}

Biodiverse natural environments not only have physical health effects and climate change adaptation potential, they also offer mental health and spiritual well-being benefits.

As an introduction to the second part of the book, Melissa Marselle provides an overview of the conceptual frameworks that provide a perspective into the ways that biodiversity can influence mental health and well-being. Coming mostly from the field of environmental psychology, the frameworks discussed are environmental preference (Biophilia Hypothesis, Preference Matrix and Fractal dimensions of nature), theories of restorative environments (Stress Reduction Theory and Attention Restoration Theory) and the Ecosystem Service Cascade Model. Each framework is 
described and its conceptualisation of biodiversity and mental well-being are detailed. Analysis of these frameworks found that no single framework details both biodiversity and mental well-being. As such, the author recommends that future researchers empirically test these frameworks using biodiversity indicators in order to further delineate which of these frameworks are 'fit for purpose' for describing the inter-relationships between biodiversity and mental well-being.

Sjerp de Vries and Robbert Snep discuss methodological issues for consideration in future biodiversity-mental health research studies. The authors point out that within studies assessing relationships between biodiversity and mental health and well-being, the concept of biodiversity is frequently adapted from its original, ecological definition. To public health and psychology researchers, a focus on species richness may imply that having more species in a habitat is always better. However, to ecologists, this interpretation has little value as they are interested in the distinct assemblages of species, including functional characteristics, or if any key species are missing. Such adaptations to the ecological definition of biodiversity, the authors argue, could result in biodiversity and mental health studies having relevance for public health and psychology, but not for nature conservation. Given that mental health promotion and nature conservation are two separate goals, the authors suggest that a more relevant research question is: can the same environment constitute a healthy, biodiverse ecosystem and enhance mental health at the same time? Suggestions are presented for future biodiversity and mental health research, with guidance for epidemiological studies assessing biodiversity in and around the residential environment on mental health and well-being. The authors recommend that future research studies should focus not on biodiversity per se, but on healthy biodiverse ecosystems that help keep people mentally healthy.

Melissa Marselle and co-authors provide a comprehensive review of the scientific literature investigating the influence of biodiversity on mental health and wellbeing. The authors present a synthesis of 24 biodiversity and mental health and well-being studies. There is some evidence to suggest that biodiversity promotes better mental health and well-being, although more studies show a non-significant effect. Due to the heterogeneity in the studies, the authors examine the pattern of results in the 24 studies by level of biodiversity (from ecosystems/habitats to single species levels), which taxonomic groups are assessed (e.g. birds, trees) and mental health or well-being outcome variables. In this way, the authors identify at which level of biodiversity, group and outcome variable non-significant effects are found. Consistent non-significant relationships were only found at the ecosystem/habitat level with mental health outcomes, as most of the other results were mixed. Clear gaps in the research were also found, as none of the 24 studies investigated the effect of perceived species richness on mental health. The researchers make several recommendations for future biodiversity and mental health and well-being studies with regard to improved, theoretically-grounded research designs, measurements of biodiversity and mental health and well-being, and investigation of mediators and dose-response relationships. 
As spiritual well-being is increasingly considered an important dimension of human health, Katherine Irvine and colleagues examine the inter-relationship between biodiversity and this aspect of human health and well-being. In their review, the authors develop an expanded understanding of spiritual well-being as encompassing one's relationships with the self, the community, the environment and transcendent Other(s), and consider this in relation to four themes from the literature. The first theme focuses on the influence of spiritual traditions on biodiversity, in which religious world views regarding nature and biodiversity can foster meaning, connection with nature, and feelings of transcendence. These experiences may result in nature conservation behaviours. The second theme, sacred places as repositories of biodiversity, highlights how spiritual values and taboos associated with specific natural sites can help to preserve biodiversity. The third theme considers the spiritual domain within ecosystems services through an examination of the measurement of spiritual well-being as a cultural ecosystem service. For the final theme, the effects of biodiversity on spiritual well-being, the authors found few empirical research studies that specifically investigated how biodiversity and biodiverse settings contribute to spiritual well-being. The authors thus examine the biodiversity-spiritual well-being relationship through an interpretation of several strands of research, for example wilderness recreation, urban green space usage, place attachment, and Attention Restoration Theory. The chapter ends with a detailed conceptual model to inform future research.

\subsection{Importance of Biodiversity, Health and Climate Change Relationships for Professionals, Practitioners and Policy-Makers}

Evidence of the health effects of biodiverse natural environments has implications for both policy and practice. This part of the book deals with the implications of the inter-relationships of biodiversity and health in the face of climate change for professionals and managers concerned with public health, nature conservation and proenvironmental behaviour, as well as how these inter-relationships are being supported by policy. Good practice examples using nature and biodiversity for human health and climate change adaptation in European countries are highlighted.

Penny Cook and co-authors discuss the implications and inter-relationships between public health, climate change and biodiversity, with specific consideration for disadvantaged groups and health inequalities. The authors provide a comprehensive overview of the numerous connections between public health and biodiversity in the face of climate change, such as: food, nutrition and water supply; environmental stress; aesthetic appreciation and spiritual well-being; socio-cultural wellbeing; physical and mental health; promotion of physical activity; and infectious diseases. From these interconnections, the authors consider the reasons why public 
health professionals should care about biodiversity loss and climate change, and support nature conservation and climate change legislation. At a local level, public health professionals could better link with local policies and practitioners to encourage greater access to and use of biodiverse urban green spaces through 'naturebased activities' like walking groups and gardening. The authors demonstrate how access to and use of natural environments can reduce social inequalities in health, a key goal of modern public health policies and programmes. However, individuals from socio-economically deprived areas are often less likely to be exposed to, and experience the benefits of, green spaces. As such, the authors highlight 'naturebased social prescriptions' as a public health intervention to facilitate contact with biodiverse natural environments for those who are less well-off in society. Specific recommendations by the authors that help implement biodiversity and climate change impacts into public health practice include linking nature conservation, public health and climate change priorities in existing local, national and international policies. In addition, working with planners and managers to ensure that green spaces are evenly distributed in urban areas is needed to avoid social inequalities in health, and robust evaluations of 'nature-based' interventions are vital in order to demonstrate causality.

Zoe Davies and co-authors discuss the impact that different nature conservation management options can have for both biodiversity and human health. The first management option, managing green spaces for people, involves no or little explicit consideration of the biodiversity quality of those spaces. These green spaces are typically in cities and designed for people rather than nature, which often results in small, isolated islands of green space that contain paved paths, recreation equipment, easy-to-maintain plants, and frequent pruning and mowing. The second management option, green spaces managed for biodiversity, involves explicit consideration of biodiversity conservation. These spaces tend to be protected areas that can be geographically distant from cities. Recreational activities of humans in protected areas are mainly managed to protect biodiversity. The authors highlight that the third management option, nature for people and nature, is rare, and discuss opportunities to manage green spaces in cities for both biodiversity and human health. For example, nature conservation professionals could work with city planners and landscape architects to add biodiversity into urban green spaces. Recommendations for managing nature for people and biodiversity from the authors include: maximizing the size of urban green spaces to sustain more species and contribute to greater health outcomes; maximizing the health benefits of nature by creating smaller urban green spaces that can be accessed and used by people; and a more international scope to understand how biodiversity and health relationships differ by cultural context.

As the consequences of climate change and biodiversity loss will require humans to change their behaviour to consume far fewer resources, Raymond De Young discusses how to initiate long-term behaviour change. This new behavioural context characterised by the necessity for fundamental change across multiple behaviours and a lack of clarity about what future behaviours will be needed - requires a differ- 
ent approach to behaviour change. The author argues for a 'capacities-first approach' to support future citizens to become 'behavioural entrepreneurs' who can identify, execute and maintain the needed behaviours themselves. Supporting behavioural entrepreneurs requires ensuring they have mental clear-headedness in order to identify, plan, self-initiate and regulate behaviour. As mental clear-headedness is a limited cognitive resource, behavioural entrepreneurs will need to spend time in nature to help restore their depleted cognitive resources and cope with the stress of living in their new world.

To embed health agendas in conservation management and vice versa, national and global policy agendas need to be aligned across the biodiversity, health and climate sectors. Horst Korn and co-authors detail these first steps and highlight the developments in the Convention on Biological Diversity (CBD) and the collaboration of CBD with the WHO on the issue of health and biodiversity. In parallel, the 2020 Health Policy Framework of the WHO European region considers environmental conditions; such considerations need to be strengthened to include specific biodiversity linkages in the next review. The UN 2030 Agenda on Sustainable Development already links various Sustainable Development Goals (SDGs) relating to health, biodiversity and climate change, and it will now depend on regional, national and local implementation to achieve its ambitious goals.

As attention on the importance of nature and health linkages increases, Hans Keune and co-authors argue that there is a need to build bridges between the nature conservation and public health sectors. Giving case-study examples of nature and health network initiatives from several European countries, the authors demonstrate how professionals from science, policy and practice can work together to address both nature and health goals. To facilitate future linkages between these sectors, the authors recommend strengthening inter-network collaboration through capacity building and integration. They additionally emphasize the need for structural support to encourage capacity-building activities. The authors stress the importance of linking existing priorities in local, national and international policies to mainstream the importance of natural environments for human health; in this regard, the One Health approach may be one way to mainstream biodiversity and health issues.

\subsection{Implications for Planning and Managing Urban Green Spaces for Biodiversity and Health in a Changing Climate}

The inter-relationships between biodiversity, human health and climate change have implications for both urban planning and management. This fourth part of the book deals with the implications of the inter-relationships of biodiversity and health in the face of climate change for protected area managers, city authorities, urban planners and landscape architects. Evaluations of urban green space interventions to 
improve human health and the environment and weaken the impact of climate change are also presented.

Kathy MacKinnon and colleagues review the ecosystem services that protected areas and NBS provide for biodiversity conservation, human health and climate change adaptation. Examining different case studies across the world, the authors illustrate how protected areas can become 'health hubs' by facilitating physical activity and stress reduction through health walks and other organized activities. As such, protected areas provide an opportunity for people to get away and experience nature and wilderness. The economic value of protected areas in cost-savings for human health, as well as climate change adaptation is examined. In order to foster the use of protected areas and NBS for both biodiversity conservation and human health, the authors recommend increased and improved collaboration between sectors and stakeholders, and propose the UN 2030 Agenda for Sustainable Development and its 20 SDGs as a mechanism for collaborative action.

Ruth Hunter and co-authors examine the environmental and human health and equity benefits of urban green space interventions. In a review of the evidence, the authors find strong support for park-based and greenway or trail interventions for encouraging physical activity and park use - but only if those interventions involved both physical changes and promotion and marketing events. There was also strong evidence that greening of vacant lots in order to improve human health and wellbeing also led to a reduction in crime. Strong evidence was also found for the environmental benefits from urban greening and roof gardens - specifically, increased biodiversity, reduced air pollution, climate change adaptation and storm water management. The authors found a lack of evidence for the impact of urban green space interventions on equity indicators. Specific recommendations for future urban green space interventions for research, policy and practice are made, such as the importance of robust evaluation research designs, economic evaluations of green space, involvement of the local community in the design of urban green spaces and using a dual approach consisting of both promotion/marketing and physical design. The authors underscore that few other public health interventions can achieve the multiple health, social and environmental benefits for all population groups that can be achieved with urban green space interventions.

As climate change imposes direct impacts on the grey, green and blue infrastructure in cities, as well as indirect impacts on the health and well-being of urban dwellers, Thomas Elmqvist and co-authors propose the concept of systems thinking to foster sustainable urban development and resilience for urban health. As a starting point, the authors argue that health should be an end goal of climate change adaptation and a proxy to examine the level of resilience of cities. The authors point out that cities are complex systems because agents from different social, ecological and technological networks connect and interact with one another at multiple scales. This complexity of different actors and networks poses enormous challenges for urban sustainability. As such, considering cities from a systems perspective - in which all actors involved in the production, sharing and use of knowledge for action are connected in a social network - can be helpful for resilience management. The 
authors maintain that such a systems perspective can lead to innovative designs of new urban infrastructure and the redesign of existing structures, such as the use of NBS to create resilience to climate change in cities in order to reduce negative health impacts and well-being.

Reflecting on the policy opportunities and challenges for considering human health in landscape planning projects, Stefan Heiland and co-authors highlight the need for increased and improved collaboration between landscape architecture, urban planning and the health sector. Examining landscape planning instruments in both Germany and the UK, the authors show how human health and biodiversity are currently considered in the design of urban green spaces through green infrastructure and ecosystem services, recreation planning, and climate change legislation. The authors conclude that health issues are implicitly touched upon or, in rare cases, explicitly named in landscape planning legislation in the UK and Germany. Consequently, the authors argue, opportunities for including health issues into landscape planning are not frequently used, and suggest this could be because health authorities are seldom involved in planning decisions. They strongly argue that including environmental interventions for health in proactive planning may reduce other hidden costs for a range of sectors, while greenspace and conservation planning cannot really go without health considerations anymore. The authors recommend legislation to overcome these disciplinary silos by requiring public health professionals to participate in urban landscape planning decisions.

\subsection{Recommendations for Research, Policy and Practice}

A number of important conclusions can be drawn from the chapters presented in this book. In this next section we identify 30 specific recommendations for research, policy and practice. These suggestions arise from those presented by the authors of this book, and incorporate recommendations debated by the European Network of Heads of Nature Conservation Agencies (ENCA) interest group on climate change at the European conference on 'Biodiversity and Health in the Face of Climate Change' held on 27-29 June 2017 in Bonn, Germany (Marselle et al. 2018).

\subsubsection{Recommendations and Challenges to Integrate Biodiversity, Health and Climate Change in Research}

Whilst there is increasing research activity to assess the linkages between biodiversity and health (Lindley et al. Chap. 2, Marselle et al. Chap. 9, Irvine et al. Chap. 10, Cook et al. Chap. 11, this volume), the chapters in this book highlight the need to expand the evidence base for the contributions of biodiversity to human health and 
well-being. In order to further this transdisciplinary field of biodiversity and health as an effective instrument for climate change adaptation, we identify key research challenges to integration:

1. Investigating biodiversity-health linkages in a changing climate: Future research should consider the potential positive and negative effects of biodiversity on human health and well-being in a changing climate. How biodiversity can help to adapt to climate pressures, e.g. through greenspace planning in urban areas or restoration of climate resilient wetlands, but also how vector-borne diseases and allergenic plants may shift in distribution with climate change, in order to identify appropriate management measures to foster positive and reduce negative health impacts.

2. Broadening research to assess the effects of biodiversity on physical, mental, spiritual and social health and well-being: Whilst there is considerable evidence of the physical health effects of biodiversity through shelter, food and medicines, there is, to date, limited evidence of the influence that biodiversity has on mental, spiritual and social well-being. This is especially relevant given that these latter health effects drove forward early conservation policy and link to intrinsic values of nature. Research should identify the influence of biodiversity on these under-investigated health and well-being outcomes.

3. Further developing theory for biodiversity-health effects: Evaluations of the impact of biodiversity on human health, or the effectiveness of nature-based interventions, require a solid theoretical basis to guide selection of health and well-being outcomes and identify causal mediators. Tending to such conceptual considerations is a necessary component for further research and integration across disciplines and sectors.

4. Identifying mechanisms: Different models have been developed to understand the various mediating pathways through which green spaces influence human health and well-being. Future research should investigate the specific mechanistic pathways through which biodiversity benefits health and well-being, for example ecosystem services, psychological restoration and perceived biodiversity. These mechanisms should be assessed through synthesis and metaanalysis of the existing literature in addition to well-designed empirical research.

5. Identifying moderators: Health effects may not be equally distributed in society and certain groups of people may experience greater health benefits from exposure to, or use of, green space and biodiverse environments. The impact of biodiversity on the health and well-being of specific socio-demographic (e.g. age and gender) and socio-economic (e.g. most disadvantaged) groups needs further scientific attention.

6. Considering 'dose-response' relationships: At present, there is a lack of knowledge on the quality and intensity of biodiversity that is required for an effect 
(how much?), the length of exposure needed before effects take place (how long?), or the duration of lasting effects for mental health and well-being. As such, future research should usefully investigate the effects of species richness, diversity and distinctiveness, the quantity of time spent in biodiverse environments, and/or the frequency of visiting a biodiverse environment that might be required for a significant change in mental health or well-being.

7. Evaluating effectiveness of interventions: Nature-based interventions need to be evaluated for their effectiveness for health and well-being, biodiversity conservation and climate change adaptation. Socio-economic factors should also be included to ensure evaluations consider potential disproportional effects across different beneficiaries. Proof of causality is important to establish when assessing the effectiveness of an intervention. Whilst randomised controlled trials may not be feasible or appropriate, nature-based interventions are complex interventions and researchers should use more robust research designs such as natural experiments, quasi-experimental before-and-after repeatedmeasures designs, or longitudinal studies (e.g. gain/loss in biodiversity or access to green space). Complex analyses such as stepped wedge, interrupted time series or structural equation modelling analyses warrant more scientific attention.

8. Analysing cost-benefits: Economic evaluations of biodiversity and interventions for human health are a significant driver for decision makers. As such, cost-benefit evaluations of the anticipated reduction in health-care costs of biodiverse green spaces are recommended. Overall, the cost-benefit analyses should be holistic, addressing all multiple benefits provided, with the specific cost reduction potential to health-care seen as just one aspect.

9. Developing models and scenarios: Scenarios and models need to be developed to investigate and forecast the human health and well-being effects of current biodiversity loss and reduced access to natural environments in a changing climate.

10. Integrating better across disciplines: By its nature, the questions considered within the field of biodiversity and health in the face of climate change are transdisciplinary and thus require integration of the natural, social and health sciences. Research should therefore be transdisciplinary in order to fully understand and measure biodiversity as well as human health impacts.

11. Increasing international scope: Current literature is geographically biased. Whilst many findings will be applicable across the Global North, we acknowledge that cultural settings matter for the appreciation of green space and more research also needs to include the Global South. As such, there is a research need to broaden understanding to include different conditions around the globe, as biodiversity-health relationships will be influenced by climate, cultural contexts and social norms. 


\subsubsection{Recommendations to Foster Wider Application of Nature-Based Solutions for Health Promotion and Climate Change Adaptation in Policy}

The chapters in this volume provide challenges and recommendations for policy. These recommendations concern two main challenge areas in increasing awareness and advancing integration across sectors and policies:

\subsubsection{Increasing Awareness recommendations of the Human Health and Well-Being Effects of Natural Environments and Biodiversity}

12. Raising awareness of multiple co-benefits: Nature-based solutions for climate change adaptation provide multiple co-benefits for human health and biodiversity. Yet policy-advisors, politicians and the public may not always be aware of these interconnections. It is thus important not only to highlight the interlinkages between climate change, human health and biodiversity but also to understand current levels of and gaps in knowledge among practitioners and policy-makers. There is an additional need to identify the type of information that would be useful to help these individuals implement actions that are based on evidence from biodiversity and health research.

13. Enhancing communication and dissemination: In order to raise awareness, communication of the health benefits of nature and biodiversity needs to be tailored to the interests of different stakeholders, practitioners and policymakers. Social media with strategic messages, brief video clips on Twitter, YouTube and other platforms as well as TV and radio are good ways to communicate and disseminate simple messages about the health benefits of biodiversity. Working with environmental charities can help disseminate these messages to larger audiences.

14. Developing manuals, guidance and tools: Manuals and guidelines for policymakers and practitioners need to be developed based on scientific evidence and good practice in applied management and policy development. Evidence and experience-based guidelines describing the key features of biodiversity required for increased health and well-being should be developed for park managers, landscape architects, urban planners and designers. Public health professionals require concrete guidance on how to use natural environments for health promotion as a complement to other already established measures. Demonstrating successful interventions or case studies where cross-sector working led to costeffective and efficient delivery of ecosystem services that provided multiple benefits will foster learning and encourage further uptake. Integrated tools of analysis and metrics from different disciplines, sectors and areas of expertise could help raise awareness and application. Building on and enhancing established decision-making process tools may be useful starting points, for example Environmental Impact Assessment and Health Impact Assessment. 


\subsubsection{Greater Integration recommendations of Biodiversity, Health and Climate Change Issues}

15. Highlighting the mutual, multiple co-benefits: Improving health and well-being and reducing harm and social inequalities are key policy priorities of governments at all levels of governance. As such, communications with decisionmakers should focus on human health and well-being as a central benefit of nature-based solutions for climate change adaptation. The co-benefits of naturebased solutions for climate change adaptation are nature conservation and enhancement of biodiversity and ecosystem services. Importantly, framing and justifying the need to protect natural environments by highlighting the enormous impact on the health of the human population, as well as delivering additional co-benefits, is more likely to be persuasive to decision-makers than a rationale based solely on conservation.

16. Building capacity: Network activities aimed at stimulating dialogue, community building and several other forms of transdisciplinary interaction between experts and stakeholders should be encouraged, as they have been shown to be successful at helping to establish cooperative working for the enhancement of biodiversity, health promotion and climate change adaptation.

17. Providing structural support: An important condition for successful networking initiatives is the availability of structural resources including supporting infrastructure. Structural support - such as financial support for cooperative networks with leadership and the support of network members and experts - is essential for cross-sectoral and cross-disciplinary working.

18. Supporting international and national policy development: To successfully introduce biodiversity and health linkages at a strategic international level, it is important to consider biodiversity, health and climate change relationships in post-2020 CBD decision-making, the implementation of the 2030 Sustainable Development Agenda, and further development of the Health 2020 policy framework of the WHO European Region. Future national, regional and global ecosystem service assessments, for example the strategic framework of a rolling work programme of the Intergovernmental Science-Policy Platform on Biodiversity and Ecosystem Services (IPBES) up to 2030 or future activities of the Mapping and Assessment of Ecosystem Services (MAES) programme, should give special attention to the health values of biodiversity and to tackling the interlinked challenges and fostering action.

19. Adopting a One Health approach to integrate biodiversity and health issues: One Health is an integrative approach, advocated by the WHO and the CBD, to address biodiversity and human health by investigating the interconnection between humans, animals, plants, agriculture, wildlife and the environment in general. The One Health approach aims to design and implement programmes, policies, legislation and research in which multiple sectors communicate and work together to achieve better public health outcomes. Policy approaches need to adopt a One Health approach, to facilitate the interlinkages of biodiversity and health in the face of climate change. 
20. Linking priorities in existing local, national and international policies: Existing policies, strategies and guidelines may, individually, address the issues of public health promotion, climate change adaptation and nature conservation. For example, health is often implicitly named in landscape and urban planning legislation when discussing climate change adaptation actions, and this provides an opportunity for linking climate change, human health and natural environment issues. Linking these existing documents and policy goals fosters a winwin, low-cost scenario in which the multiple co-benefits for human health and biodiversity conservation can be achieved. Public health leaders should work with governments, planners and ecologists to ensure that health considerations are incorporated into national and local planning and development regulations as well as environment and sustainability strategies and action plans.

21. Linking to United Nations Sustainable Development Goal (SDG) indicators: The 2030 Agenda for Sustainable Development and its 20 SDGs present a framework for collaborative action to respond to a range of global challenges that cannot be solved in isolation. SDGs relating to biodiversity and health in the face of climate change are SDG 3 'good health and well-being', SDG 11 'sustainable cities and communities', SDG 13 'climate action', SDG 14 'life below water' and SDG 15 'life on land'. To achieve the multiple aims of the SDGs, it will be important to work across sectors to protect, manage and restore the biodiversity and ecosystem services that contribute to human well-being, and reduce the impacts of climate change. SDGs provide a focus on a specific challenge to monitor progress, success and sustainability. They can also guide regional, national and local policies and practices.

22. Fostering continued dedication to climate change agreements: In order to combat the impact that climate change will have on human health and biodiversity, it is important to ensure continued commitment to existing international policy accords. As such, it is paramount that nations adhere to the climate change mitigation policies under the 2015 Paris Agreement. Linking these climate changefocused policies to the health agenda will help to create alliances and innovative implementation and funding schemes.

\subsubsection{Recommendations to Implement Existing Knowledge into Practice}

We know enough to act now. The chapters in this volume provide good practice case studies that demonstrate how research informs implementation of nature-based solutions to foster human health in the face of climate change. We identify two key challenges with regards to design and planning as well as management for integrating biodiversity and health issues when addressing climate change adaptation in practice: 


\subsubsection{Design and Planning recommendations to Enhance Contact with and Experience of Nature and Biodiversity}

23. Designing in biodiversity: Landscape architects should be encouraged to 'design in biodiversity' by fostering native plants and wildlife in public parks or conservation areas as well as in the urban matrix. This increases the opportunities for people to interact with biodiversity and obtain its health benefits, whilst enhancing biodiversity conservation and also contributing to climate change adaptation.

24. Creating a mixture of 'everyday' green spaces: It is important for people to have contact with natural environments in their daily life (e.g. on their way to school or work, around the home). Various urban green spaces (ranging from street trees, 'pocket parks' and green school yards to larger urban parks) should be created to increase the opportunities for people to be exposed to biodiversity for their own health and well-being. To use green spaces for health promotion, city planners should create publicly accessible green spaces that are evenly distributed across the spatial extent of towns and cities; this may be mandated in urban planning guidelines. In addition, urban green spaces can contribute significantly to adaptation to climate change.

25. Creating 'green' corridor connections: Cities should be planned to include 'green corridors' through which citizens can travel from smaller urban green spaces to larger green spaces or protected areas. These 'green corridors' create additional opportunities for recreation and restoration, which have health, well-being and social benefits. Further, 'green corridors' can contribute to biodiversity conservation by increasing the amount of green space and providing links between different habitats for migration and sustaining metapopulations of species. In addition, green corridors can serve as important avenues for fresh air.

26. Promoting and managing protected areas as 'health hubs': Protected areas provide opportunities for nature conservation as well as human health benefits. Thus, protected areas have the potential to be 'health hubs' for both nature and people. To encourage use, social interventions, such as guided health walks, can be used to highlight the value that a protected area delivers for human health and well-being. Such positive nature experiences can deepen people's commitment to conserve natural spaces and support protected areas. Dedicated management is needed in order to offer natural health services to humans in protected areas whilst protecting biodiversity.

27. Co-designing with stakeholders: The needs of the local community and other stakeholders must be taken into consideration in order to build ownership, cooperation and collaboration on biodiversity, health and climate change issues. A co-designed framework plan for biodiversity, health and climate change strategies and management is likely to be the most successful. 


\subsubsection{Management Recommendations to Improve the Use of Urban Green Spaces}

28. Utilising physical interventions: Access to a green space does not necessarily result in its use. Physical design and management can improve the biodiversity quality and aid the use of green spaces. Physical interventions to facilitate use involves considering the needs of different users in the local community as well as long-term health, social and environmental effects. Management plans for green spaces should ensure these spaces are maintained in order to avoid perceptions of neglect, as overgrowth and/or broken benches/play structures/rubbish can increase fear of crime and reduce use.

29. Employing social interventions: To further encourage use, promotion and marketing events should be used in combination with physical interventions. It is especially important to target interventions to individuals in socially-deprived neighbourhoods. Practitioners should use nature-based social-prescribing interventions, such as health walks in forests, conservation volunteering or therapeutic gardens, to encourage use of and contact with biodiverse green spaces.

30. Monitoring impact: In order to develop evidence of impact and economic value, it is important to implement robust monitoring and evaluation of the effect of nature-based solutions on climate change adaptation, human health and well-being, and biodiversity. This will help to advance both management and policy in the interconnected field of biodiversity, health and climate change.

\subsection{Outlook}

Facing global challenges, we need concerted action to foster human health and biodiversity, the foundation of life. It is time to act now and to urgently address increasing health issues and to harness NBS to health promotion. In a changing climate the importance for nature-based solutions for human health will increase. In the long run modern combinations of nature-based solutions with technical solutions will be the cheaper alternative in comparison with choosing technical solutions on their own. Nature-based solutions have additional advantages in that they can pose win-win-win solutions for biodiversity, human health and adaptation to climate change, and their management actions are more easily reversible and adaptable.

In international policy, practice and research, the issue of biodiversity and human health, with a link to climate change as a major stressor for both, is high on the agenda. Research is focusing attention on this topic with new transdisciplinary research programmes. Since climate change will exacerbate societal problems with respect to health, policy needs to act now to put scientific evidence into real action. We hope this volume provides a critical overview and evaluation of the interlink- 
ages of climate, health and biodiversity, and will inform and trigger further policy development and practical implementation, as well as stimulate ongoing scientific debate and open innovative research avenues.

Acknowledgements We are grateful to the ENCA interest group on climate change for initial discussions in June 2017. This work was supported by the German Federal Agency for Nature Conservation (BfN) with funds from the German Federal Ministry for the Environment, Nature Conservation, and Nuclear Safety (BMU) through the research project 'Conferences on Climate Change and Biodiversity' (BIOCLIM, project duration from 2014 to 2017, funding code: 351480 020A). Dr. Irvine's involvement was funded by the Rural and Environment Science and Analytical Services Division of the Scottish Government.

\section{References}

Aerts R, Honnay O, Van Nieuwenhuyse A (2018) Biodiversity and human health: mechanisms and evidence of the positive health effects of diversity in nature and green spaces. Br Med Bull 127(1):5-22

Bellard C, Bertelsmeier C, Leadley P, Thuiller W, Courchamp F (2012) Impacts of climate change on the future of biodiversity. Ecol Lett 15:365-377

Cox DT, Shanahan DF, Hudson HL et al (2017) Doses of neighborhood nature: the benefits for mental health of living with nature. Bioscience 67:147-155

Dallimer M, Irvine KN, Skinner AMJ et al (2012) Biodiversity and the feel-good factor: understand associations between self-reports human well-being and species richness. Bioscience 62(1):47-55

Fuller RA, Irvine KN, Devine-Wright P et al (2007) Psychological benefits of greenspace increase with biodiversity. Biol Lett 3:390-394

Hartig T, Mitchell R, de Vries S, Frumkin H (2014) Nature and health. Annu Rev Public Health 35:207-228

Lovell R, Wheeler BW, Higgins SL et al (2014) A systematic review of the health and wellbeing benefits of biodiverse environments. J Toxicol Environ Health B Crit Rev 17(1):1-20

Markevych I, Schoierer J, Hartig T et al (2017) Exploring pathways linking greenspace to health: theoretical and methodological guidance. Environ Res 158:301-317

Marselle MR, Irvine KN, Warber SL (2014) Examining group walks in nature and multiple aspects of well-being: a large-scale study. Ecopsychology 6:134-147

Marselle M, Stadler J, Korn H, Bonn A (eds) (2018) Proceedings of the European conference "Biodiversity and Health in the Face of Climate Change - Challenges, Opportunities and Evidence Gaps". BfN-Skripten 509. Federal Agency for Nature Conservation, Germany, Bonn. https://www.bfn.de/fileadmin/BfN/service/Dokumente/skripten/Skript509.pdf

Potschin MB, Haines-Young RH (2011) Ecosystem services: exploring a geographical perspective. Prog Phys Geogr 35:575-594

Shanahan DF, Fuller RA, Bush R et al (2015) The health benefits of urban nature: how much do we need? Bioscience 65:476-485

Steffen W, Richardson K, Rockström J et al (2015) Planetary boundaries: guiding human development on a changing planet. Science 347:736-747

van den Bosch M, Ode Sang $\AA$ (2017) Urban natural environments as nature-based solutions for improved public health - a systematic review of reviews. Environ Res 158:373-384

WHO (2017a) Fact sheets on sustainable development goals: health targets for noncommunicable diseases. World Health Organization Regional Office for Europe, Copenhagen, Denmark. http://www.euro.who.int/__data/assets/pdf_file/0007/350278/Fact-sheet-SDG-NCDFINAL-25-10-17.pdf?ua=1. Accessed 30 July 2018 
WHO (2017b) Noncommunicable diseases. World Health Organization Regional Office for Europe, Copenhagen, Denmark. http://www.euro.who.int/en/health-topics/noncommunicablediseases. Accessed 13 July 2018

WHO \& CBD (2015) Connecting global priorities: biodiversity and human health. A state of the knowledge review. World Health Organization \& Secretariat of the Convention on Biological Diversity. http://www.who.int/globalchange/publications/biodiversity-human-health/en/. Accessed 30 July 2018

Open Access This chapter is licensed under the terms of the Creative Commons Attribution 4.0 International License (http://creativecommons.org/licenses/by/4.0/), which permits use, sharing, adaptation, distribution and reproduction in any medium or format, as long as you give appropriate credit to the original author(s) and the source, provide a link to the Creative Commons license and indicate if changes were made.

The images or other third party material in this chapter are included in the chapter's Creative Commons license, unless indicated otherwise in a credit line to the material. If material is not included in the chapter's Creative Commons license and your intended use is not permitted by statutory regulation or exceeds the permitted use, you will need to obtain permission directly from the copyright holder.

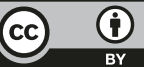

